\title{
Modeling, Simulation, and Stabilization of a Two- Wheeled Inverted Pendulum Robot Using Hybrid Fuzzy Control
}

\author{
Made Rahmawaty \\ Jurusan Teknologi Industri, Teknik Mekatronika Politeknik Caltex Riau, Riau \\ Jl. Umbansari No 1 Rumbai, Pekanbaru, Riau, 28265, Indonesia
}

\section{Article Info \\ Article History: \\ Received July 20, 2020 \\ Revised August 14, 2021 \\ Accepted August 16, 2021}

\section{Keywords: \\ Fuzzy T-S, \\ Fuzzy Mamdani \\ Parallel Distributed \\ Compensation (PDC) \\ Pole Placement \\ Two Wheels Inverted \\ Pendulum}

\begin{abstract}
Two wheels inverted pendulum robot has the same characteristics as an inverted pendulum, which are unstable and nonlinear. Nonlinear systems can often be linearized by approximating them by a linear system obtained by expanding the nonlinear solution in a series, and then linear techniques can be used. Fuzzy logic control is the famous nonlinear controller that researchers have used to analyze the performance of a system due to the easiness of understanding the nature of the controller. This research discusses two wheels inverted pendulum robot design using hybrid fuzzy control. There are two types of fuzzy control: Fuzzy Balanced Standing Control (FBSC) to maintain stability and Fuzzy Traveling and Position Control (FTPC). Based on Takagi-Sugeno (T-S) fuzzy model on two wheels inverted pendulum robot, FBSC control used Parallel Distributed Compensation (PDC) with pole placement technic. Based on two wheels inverted pendulum robot movement characteristics, FTPC was designed using Mamdani Fuzzy architecture. FTPC control is used to help FBSC maintain robot stability and adjust to the desired position. The simulation result shows that the controller for two wheels inverted pendulum robot can stabilize pendulum angle in 0 radian and close to the desired position.
\end{abstract}

Corresponding Author:

Made Rahmawaty

made@pcr.ac.id

Jurusan Teknologi Industri, Teknik Mekatronika

Politeknik Caltex Riau
This work is an open-access article and licensed under a Creative Commons Attribution-ShareAlike 4.0 International License (CC BY-SA 4.0).

\section{INTRODUCTION}

Two wheels inverted pendulum robot is a robot that has 2 (two) wheels that work like a reversed pendulum. This robot has the same characteristics as the inverted pendulum, which are unstable and nonlinear. The main goal of this research is to stabilize the robot to its reversed position and back to its previous position or desired position.

In recent years, fuzzy control design based on TakagiSugeno (T-S) model has been widely used on more comprehensive applications such as manipulator robots [1], trailers [2], hovercraft [3], and helicopters [4]. Fuzzy control had been well implemented in so many nonlinear systems. However, systematically, fuzzy control design for system stability analysis is still an interesting challenge, especially in nonlinear system control design. This is because nonlinearity is always interfering with real system control since it is only partially known, it is hard to describe, and there are only a few measured states [5].

There have been several studies on two-wheeled inverted pendulum robots with fuzzy control, among others, those conducted by Almeshal et al., who used robust hybrid fuzzy logic control with movable loads [6], Kharola's research made a control design using the fuzzy method. Logic control strategy often is used to control and stabilize the robot [7]. Furthermore, [8] the author developed a fuzzy logic controller for the twowheeled LEGO EV3 robot. Finally, the research conducted by Huang et al. designed a type- 2 interval fuzzy logic modeling and inverted pendulum control two-wheel moving [9].

This research proposed a design and simulation of two wheels inverted pendulum robot using hybrid fuzzy control. The 


\section{Indonesian Journal of Electronics, Electromedical Engineering, and Medical Informatics (IJEEEMI) \\ IJEEMI, Vol. 3, No. 3, August 2021, pp. 93-98}

DOI: 10.35882/ijeeemi.v3i3.2

controller design has two fuzzy controls, namely fuzzy balanced standing control (FBSC) and fuzzy traveling and position control (FTPC). FBSC uses parallel distributed compensation (PDC) concept with pole placement technic to stabilize the robot based on fuzzy Takagi-Sugeno. At the same time, FTPC uses Mamdani architecture's heuristic rule to design movement and position. The robot is a nonlinear model and needs to be changed to a linear model via a linearization process.

This paper is organized into five sections. Section I contains an introduction to this research. The following section discusses the mathematical model of two wheels inverted pendulum robot and hybrid fuzzy control. Section III explains the result of the two wheels inverted pendulum robot, while section IV discusses the limitation of the results and compares the results with others. Lastly, the conclusion for this research is stated in section V.

\section{METHOD AND MODELING}

\section{A. Mathematical Model of Two Wheels Inverted Pendulum Robot}

There are two steps to obtain a mathematical model of two wheels inverted pendulum robot. First is knowing the coordinate system used by two wheels inverted pendulum robot with geometric parameters shown in Fig. 1. $\phi$ is pendulum tilt angle, and $\theta$ is the average value of motor rotary angle, described as follows $\theta=\left(\theta_{R}+\theta_{L}\right) / 2$. $\theta_{R}$ and $\theta_{L}$ is the rotary angle of left motor and right motor respectively. The geometric parameters and values of a two-wheeled inverted pendulum robot are presented in Table 1 [10] [11] [12]. Based on the concept of physic, the force resultant of the $\mathrm{x}$-axis is described in equation (1) [13].

$$
\left(M_{p}+M_{c}\right) r \ddot{\theta}+M_{p} l \ddot{\phi} c \quad \phi=f_{x}
$$

where $f_{x}$ does motor rim give force along the $\mathrm{x}$-axis, described as $f_{x}=f_{R}+f_{L}$, where $f_{R}$ and $f_{L}$ describes the forces of the rim of the right motor and left motor, respectively, the momentum on the pendulum circle point describes equation (2) [14].

$$
\begin{aligned}
& M_{p} l \imath \ddot{\theta} c_{1} \quad+M_{p} l^{2} \ddot{\phi}-M_{p} g=0 \\
& \ddot{\theta}=\frac{M_{p} g-M_{p} l^{2} \ddot{\phi}}{M_{p} l_{1}}=\frac{g \quad-l \ddot{\phi}}{r_{1}}
\end{aligned}
$$

While the torque is described in equation (3).

$$
\begin{aligned}
& u_{f}(t)=f_{x}(t) r \\
& \left(M_{p}+M_{c}\right)\left\{\frac{g s \cdot \phi-l \ddot{\phi}}{c \cdot \phi}\right\}+M_{p} l \ddot{\phi} c_{l} \quad \phi=\frac{u_{f}(t)}{r}
\end{aligned}
$$

Two wheels inverted pendulum robot consists of 4 states that described as a vector $x(t)=\left[\begin{array}{llll}\phi & \dot{\phi} & \dot{\theta} & \theta\end{array}\right]^{T}$ where $x_{1}=\phi$ declares pendulum tilt angle, $x_{2}=\dot{\phi}$ declares pendulum angle speed which is the first derivative of pendulum tilt angle $\left(x_{2}=\right.$ $\left.d x_{1} / d\right), x_{3}=\dot{\theta}$ declares motor rotational angle speed, which is the first derivative of motor rotational angle $(d / d)$, and $x_{4}=\theta$ declare motor rotational angle. The following is two wheels inverted pendulum robot state equation (4).

$$
\begin{aligned}
& \dot{x}_{1}=x_{2} \\
& \dot{x}_{2}=\frac{\left(M_{p}+M_{C}\right) g x_{1}}{\left.l\left(1-\beta^{2}\right) M_{p}+M_{C}\right\}}-\frac{u_{f}(t) \beta}{r\left\{\left(1-\beta^{2}\right) M_{p}+M_{c}\right\}} \\
& \dot{x}_{3}=\frac{u_{f}(t)}{\left\{\left(1-\beta^{2}\right) M_{p}+M_{c}\right\} r^{2}}-\frac{M_{p} g x_{1} \beta}{\left\{\left(1-\beta^{2}\right) M_{p}+M_{c}\right\} r} \\
& \dot{x}_{4}=x_{3}
\end{aligned}
$$

Where;

$$
\begin{aligned}
& \alpha=\frac{s}{\phi} \phi \\
& \beta=c_{1} \phi
\end{aligned}
$$

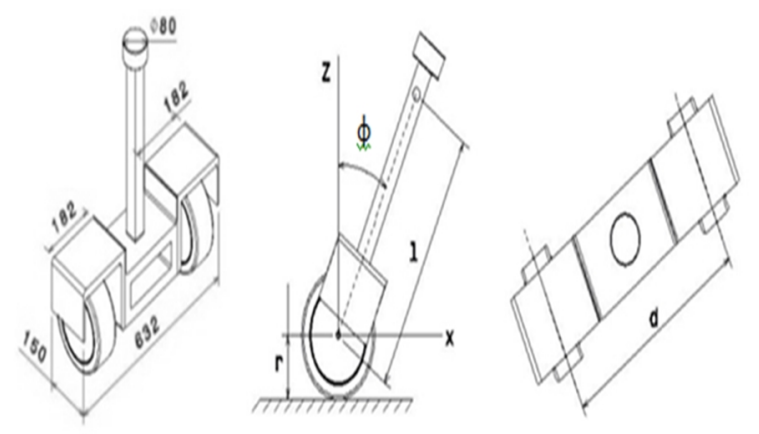

Fig. 1. Dynamic Model of Two Wheels Inverted Pendulum Robot [6].

TABLE I. GEOMETRIC PARAMETERS AND VALUES OF A TWO-WHEELED INVERTED PENDULUM ROBOT [10]

\begin{tabular}{l|c|c|c}
\multicolumn{1}{c|}{ Parameter } & Symbol & Value & Unit \\
\hline Pendulum mass & $M_{p}$ & 9.1 & {$[K]$} \\
\hline Train Mass & $M_{C}$ & 25.2 & {$[K]$} \\
\hline $\begin{array}{l}\text { The length between the } \\
\text { wheel shaft and the center } \\
\text { of gravity of the pendulum }\end{array}$ & $l$ & 0.5 & {$[m]$} \\
\hline Wheel radius & $r$ & 0.1 & {$[m]$} \\
\hline $\begin{array}{l}\text { Distance between left and } \\
\text { right wheels }\end{array}$ & $D$ & 0.44 & {$[m]$} \\
\hline Gravitational acceleration & $g$ & 9.8 & {$\left[m / s^{2}\right]$} \\
\hline
\end{tabular}

B. Hybrid Fuzzy Control on Two Wheels Inverted Pendulum Robot

1) Mamdani Fuzzy Controller Design

FTPC controller for the position of the two wheels inverted pendulum robot is using Mamdani Fuzzy model with two premises and 49 if-the rules. This control design is used not only 
to help the FBSC controller maintain robot stability but also to guide the desired position. At the initial condition, a positive or negative direction value is assigned to the pendulum angle, then the two wheels inverted pendulum robot will move forward or backward, preventing the pendulum from falling and keeping the robot walking through to the desired position. If an error occurs, the two wheels inverted pendulum robot position describes in equation (5).

$$
e(t)=p(t)-p_{d}
$$

where $p_{d}$ declares the two wheels inverted pendulum robot desired position and $p(t)$ declares the two wheels inverted pendulum robot current position, so the desired state will be $x_{d}(t)=\left[\begin{array}{llll}\phi_{d}(t) & 0 & 0 & 0\end{array}\right]^{T}$, while state error $z(t)$ describes equation (6).

$$
\begin{aligned}
z(t) & =x(t)-x_{d}(t) \\
z(t) & =\left[\begin{array}{lll}
\phi(t)-\phi_{d}(t) \dot{\phi}(t) \dot{\theta}(t) & \theta(t)
\end{array}\right] \\
& =\left[\begin{array}{lll}
e_{d}(t) \dot{\phi}(t) & \dot{\theta}(t) & \theta(t)
\end{array}\right]
\end{aligned}
$$

where $e_{d}(t)$ declares pendulum angle error.
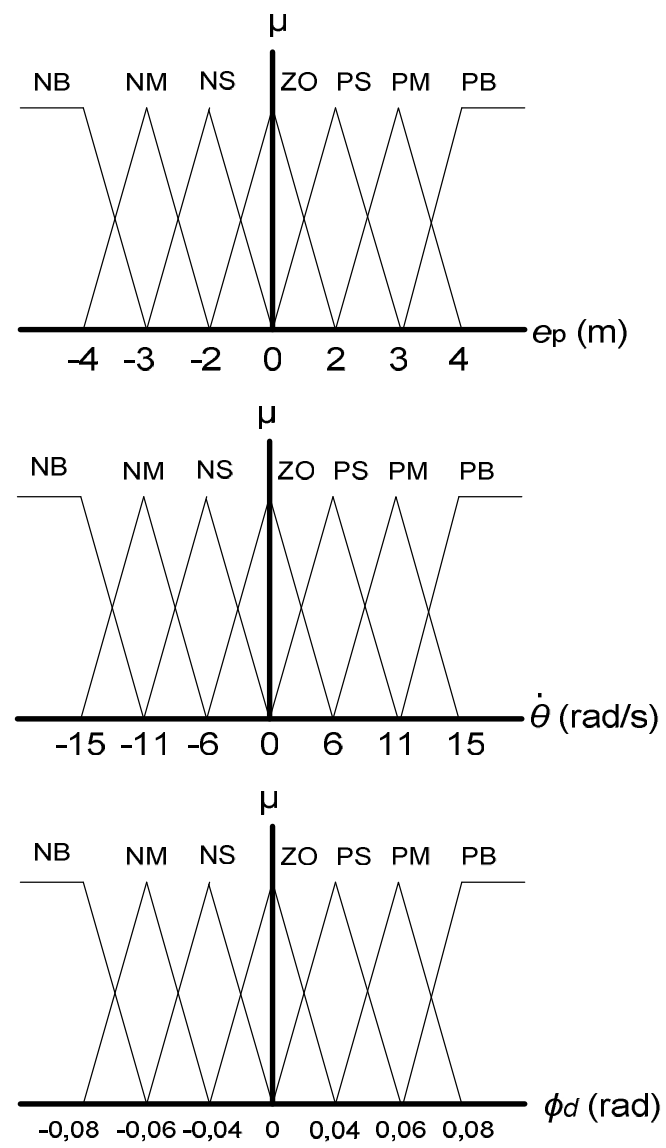

Fig. 2. Membership Function FTPC Controller
Membership function used in $e_{p}, \dot{\theta}$, and $\phi_{d}$ are triangle and described as follows (7):

$$
\mu(x)=f(x ; a, b, c)=\left\{\begin{array}{cc}
0, & x \leq a \\
\frac{x-a}{b-a}, & a \leq x \leq b \\
\frac{c-x}{c-b}, & b \leq x \leq c \\
0, & c \leq x
\end{array}\right.
$$

There are 7 (seven) fuzzy sets used in $e_{p}, \dot{\theta}$, and $\phi_{d}$, namely, negative big (NB), negative medium (NM), negative small (NS), zero (ZO), positive small (PS), positive medium (PM), and positive big (PB). If consequence " $u$ is $\phi_{d}$ " is a conclusion obtained when premises " $x$ is $e_{p}$ " and " $x$ is $\dot{\theta}$ " are fulfilled, then there are 49 fuzzy rules for robot control position shown in Table 2. Fig. 2 depicts membership function with two inputs

\begin{tabular}{|c|c|c|c|c|c|c|c|c|}
\hline \multirow{2}{*}{\multicolumn{2}{|c|}{$\begin{array}{c}\text { Consequence } \\
\phi_{d}\end{array}$}} & \multicolumn{7}{|c|}{$\begin{array}{c}\text { Premise } \\
\dot{\theta}\end{array}$} \\
\hline & & NB & NM & NS & ZO & PS & $\mathbf{P M}$ & PB \\
\hline \multirow{7}{*}{$\begin{array}{c}\text { Premise } \\
e_{p}\end{array}$} & NB & PB & $\mathrm{PB}$ & PB & $\mathrm{PB}$ & $\mathrm{PM}$ & PS & $\mathrm{ZO}$ \\
\hline & NM & PB & PB & PB & PM & PS & $\mathrm{ZO}$ & NS \\
\hline & NS & PB & PB & $\mathrm{PM}$ & PS & $\mathrm{ZO}$ & NS & NM \\
\hline & $\mathrm{ZO}$ & PB & $\mathrm{PM}$ & PS & $\mathrm{ZO}$ & NS & $\mathrm{NM}$ & NB \\
\hline & PS & PS & PS & $\mathrm{ZO}$ & NS & NM & NB & NB \\
\hline & PM & PS & $\mathrm{ZO}$ & NS & NM & NB & NB & NB \\
\hline & PB & $\mathrm{ZO}$ & NS & NM & NB & NB & NB & NB \\
\hline
\end{tabular}
(premises), i.e., position error $\left(e_{p}\right)$ and wheel rotation speed $(\dot{\theta})$ also output (consequence) shown as pendulum desired angle $\left(\phi_{d}\right)$.

TABLE II. FUZZY RULE TABLE FOR FTPC CONTROL

2) Fuzzy Takagi-Sugeno Controller Design

Fuzzy T-S model design for balancing the two wheels inverted pendulum robot is using 2 rules. Membership function in this fuzzy rule only implemented for pendulum angle output $(\phi)$. Membership function in this research is triangle membership function describes in equation (8).

$\mu(x)=f(x ; a, b, c)=\left\{\begin{array}{cc}0, & x \leq a \\ \frac{x-a}{b-a}, & a \leq x \leq b \\ \frac{c-x}{c-b}, & b \leq x \leq c \\ 0, & c \leq x\end{array}\right.$

where $a$ is the left limit parameter, $b$ is the midpoint and $c$ is the right limit, while $x$ is a variable.

\section{Accredited by Ministry of Research and Technology /National Research and Innovation Agency, Indonesia Decree No: 200/M/KPT/2020}


This research is conducted using two rules. The first rule is used when the angle is 0 radian, and the second is when the angle is \pm 0.2 radian so that the membership grade can be depicted in Fig. 3.

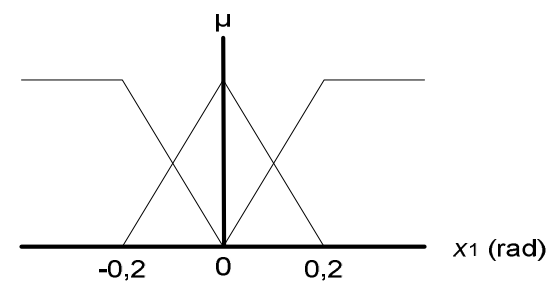

Fig. 3. Pendulum Angle Membership Function.

There are two kinds of rules used: rule for plant and rule for the controller. Rules for the plant are:

The first rule for plants:

$$
\begin{gathered}
\text { if } x_{1} \text { is around } 0 \\
\tau \eta \varepsilon v \dot{x}_{1}(t)=A_{1} x(t)+ \\
B_{1} u(t)
\end{gathered}
$$

The second rule for plants:

$$
\text { if } x_{1} \text { is around } 0.2
$$

$$
\tau \eta \varepsilon v \dot{x}_{2}(t)=A_{2} x(t)+B_{2} u(t)
$$

Henceforth, Parallel Distributed Compensation (PDC) is used to specify controller parameter state $z(t)$ in equation (6). Control rules in PDC designed based on corresponding plant rule in equation (9) are specified as follows:

rule 1 for controller:

$$
\begin{aligned}
& \text { if } x_{1} \text { is around } 0 \\
& \tau \eta \varepsilon \vee \\
& \tau=-K_{1} z(t)
\end{aligned}
$$

rule 2 for controller:

$$
\begin{aligned}
& \text { if } x_{1} \text { is around } 0,2 \\
& \tau \eta \varepsilon \vee u=-K_{2} z(t)
\end{aligned}
$$

The result from all control signal written as an equation 11 .

$$
u=-\left(\mu_{1} K_{1}+\mu_{2} K_{2}\right) \mathrm{z}(t)
$$

where $\mu_{1}$ is the weight of rule 1 and $\mu_{2}$ is the weight of rule 2 .

Block diagram of two wheels inverted pendulum robot using hybrid fuzzy control is depicted in Fig. 4.

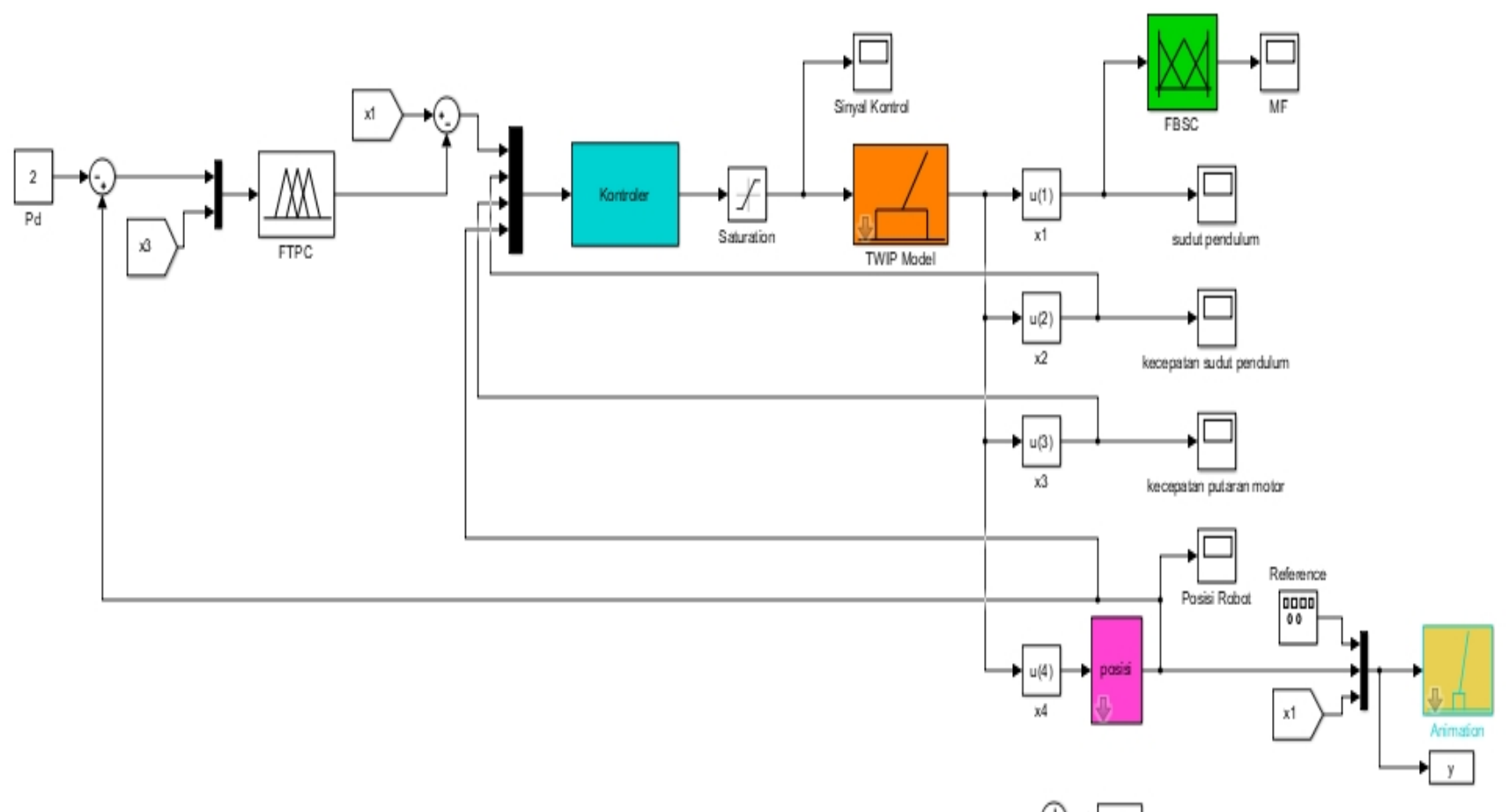

Fig. 4. Two Wheels Inverted Pendulum Robot System Block Diagram. 


\section{Indonesian Journal of Electronics, Electromedical Engineering, and Medical Informatics (IJEEEMI) \\ IJEEMI, Vol. 3, No. 3, August 2021, pp. 93-98}

\section{RESULTS}

In this part, the designed controller is simulated on two wheels inverted pendulum robot plant. The robot system statespace equation is obtained in equation (12) based on equation four linearizations.

$$
\dot{x}(t)=A_{i} x(t)+B_{i} u(t) \quad, i=1,2
$$

With working point $x_{1}$ is $0 ; 0,2 A_{i}$ dan $B_{i}$ are then obtained as follows respectively:

$$
\begin{aligned}
& A_{1}=\left[\begin{array}{cccc}
0 & 1 & 0 & 0 \\
26.6778 & 0 & 0 & 0 \\
-35.3889 & 0 & 0 & 0 \\
0 & 0 & 1 & 0
\end{array}\right] \text { and } B_{1}=\left[\begin{array}{c}
0 \\
-0.7937 \\
3.9683 \\
0
\end{array}\right] \\
& A_{2}=\left[\begin{array}{cccc}
0 & 1 & 0 & 0 \\
25.0541 & 0 & 0 & 0 \\
-31.1953 & 0 & 0 & 0 \\
0 & 0 & 1 & 0
\end{array}\right] \text { and } B_{2}=\left[\begin{array}{c}
0 \\
-0.7669 \\
3.9125 \\
0
\end{array}\right]
\end{aligned}
$$

With pole placement areas at $-2.7 ;-2.72 ;-2.9 ;-2.91$ and 2.8 ; $-2.81 ;-3.0 ;-3.1$, then feedback gain values are:

$$
\begin{aligned}
& K_{1}=\left[\begin{array}{llll}
-97.1624 & -19.8332 & -1.1367 & -0.7968
\end{array}\right] \\
& K_{2}=\left[\begin{array}{llll}
-104.7158 & -22.1657 & -1.3518 & -0.9875
\end{array}\right]
\end{aligned}
$$

Fig. 5 is the simulation result based on state feedback gain control algorithm for initial angle 0.2 radian and initial angle 0.3 radian with pendulum angle speed, pendulum rotary angle, motor rotary angle, and robot position is 0 .

Peak time is used to measure robot position performance, and pendulum angle is determined when it reaches the highest point. Fig. 5 shows the performance measure for robot position compared to initial angle 0.2 radian and initial angle 0.3 radian with the robot position is back to the initial position. Peak time at initial angle 0.2 radian is around 0.61 second with maximum overshoot is around 1.465 meters, and settling time is around 5.91 seconds, while peak time at initial angle 0.3 radian is around 0.72 second with maximum overshoot is around 2.053 meters, and settling time is around 6.29 seconds.

Fig. 5 also shows that the pendulum robot is experiencing deviation before moving back to its original position. At the initial angle of 0.3 radians, the robot experiences the largest deviation compared to when the initial angle is 0.2 radians. It is to be concluded that the larger the given initial angle, the larger the peak time and the maximum overshoot.

Fig. 6 depicts control signal performance measurement comparing initial angle 0.2 radian against initial angle 0.3 radian. The generated control signal is 20 Newton meters.

Pendulum angle performance is shown in Fig. 7. Peak time at initial angle 0.2 radian is around 0.61 second with maximum undershoot is around 0.008755 radian, and settling time is around 1.83 seconds, while peak time at initial angle 0.3 radian is around 0.81 second with maximum undershoot is around
0.01215 radian, and settling time is around 1.86 second. Based on the responses, it can be concluded that the larger the initial angle, the larger the undershoot value.

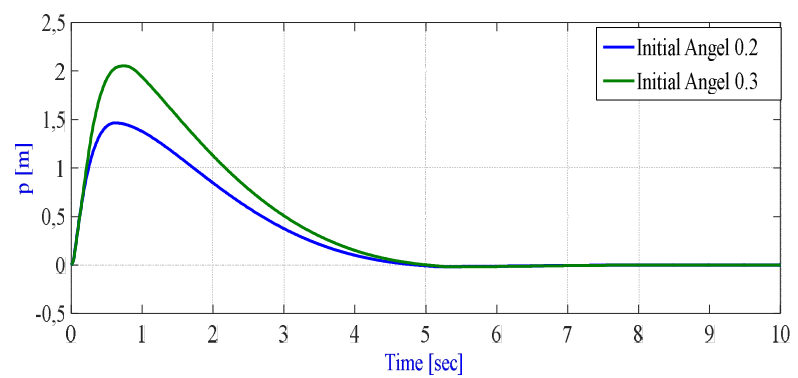

Fig. 5. Robot Position Response Simulation Comparison Result

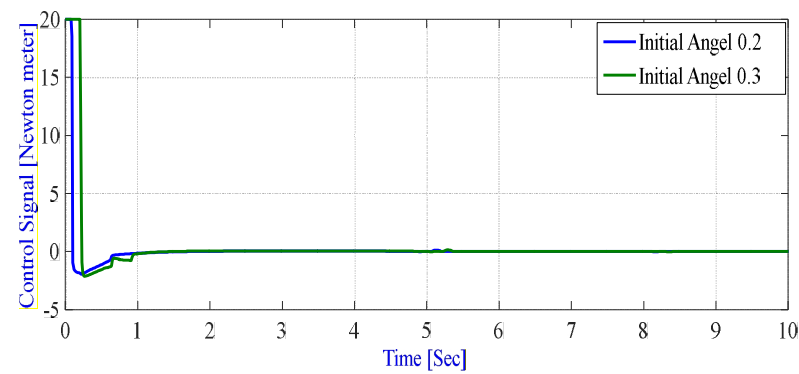

Fig. 6. Control Signal Response Simulation Comparison Result.

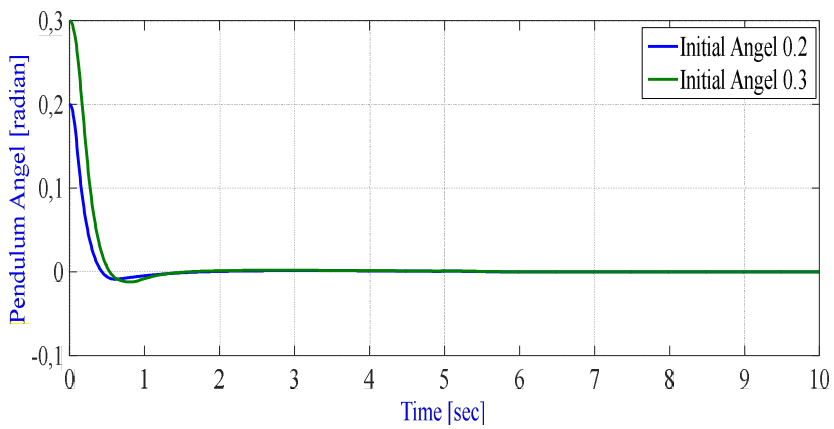

Fig. 7. Pendulum Angle Response Simulation Comparison Result

The following is examination simulation using initial angle 0.2 radian with $p_{d}=0.2$ meter and $p_{d}=0.4$ meter. Fig. 8 depicts performance measurement for robot position with initial angle 0.2 radian and $p_{d}=0.2$ meter, peak time is around 0.65 second, with maximum overshoot is around 1.554 meter, settling time is around 7.67 seconds and robot position 0.1847 meter.

Pendulum angle response can be seen in Fig. 9, with peak time when initial angle 0.2 radian is around 0.61 second with maximum undershoot is around 0.0102 radian, and settling time is around 2.08 seconds.

Simulation in Fig. 10 with initial angle 0.2 radian and $p_{d}=$ 0.4 meter. Peak time when the initial angle 0.2 radian is around 0.69 second with maximum overshoot is around 1.7 meters, and settling time is around 7.2 seconds and robot position 0.3679 meter.

\section{Accredited by Ministry of Research and Technology /National Research and Innovation Agency, Indonesia Decree No: 200/M/KPT/2020}




\section{Indonesian Journal of Electronics, Electromedical Engineering, and Medical Informatics (IJEEEMI) \\ IJEEMI, Vol. 3, No. 3, August 2021, pp. 93-98 \\ DOI: 10.35882/ijeeemi.v3i3.2}

Pendulum angle performance is shown in Fig. 11 with initial angle 0.2 radian, peak time when initial angle 0.2 radian is around 0.62 second with maximum undershoot is around 0.01269 radian, and settling time is around 2.14 seconds.

Based on the available data and compare it with the error obtained from the simulation result against the desired position, it can be concluded that the greater the desired position $\left(p_{d}\right)$, the greater the position error. When $p_{d}=0.2$ meter, position error is $7.65 \%$, while when $p_{d}=0.4$ meters, the position error is $8.02 \%$.

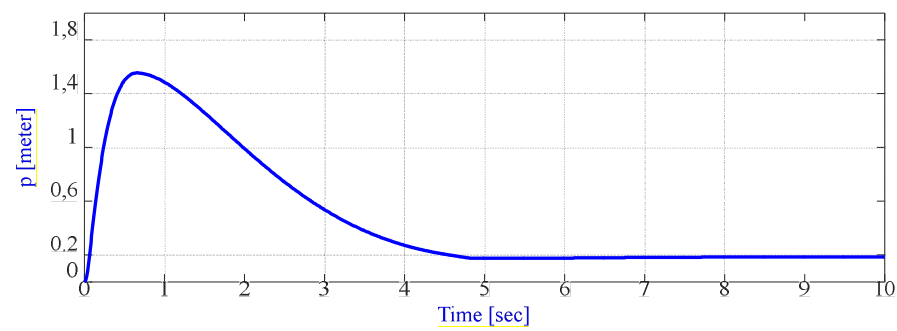

Fig. 8. Result of Robot Position Response Simulation with Initial Angle 0.2 radian and $\boldsymbol{p}_{\boldsymbol{d}}=0.2$ meter.

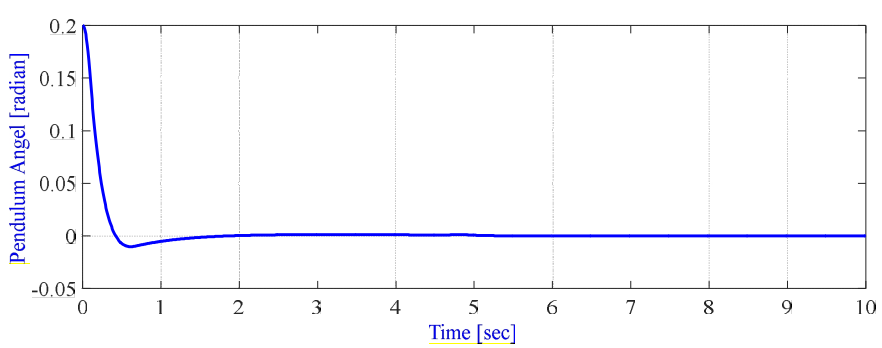

Fig. 9. Result of Pendulum Angle Response Simulation with Initial Angle 0.2 radian and $\boldsymbol{p}_{\boldsymbol{d}}=0.2$ meter.

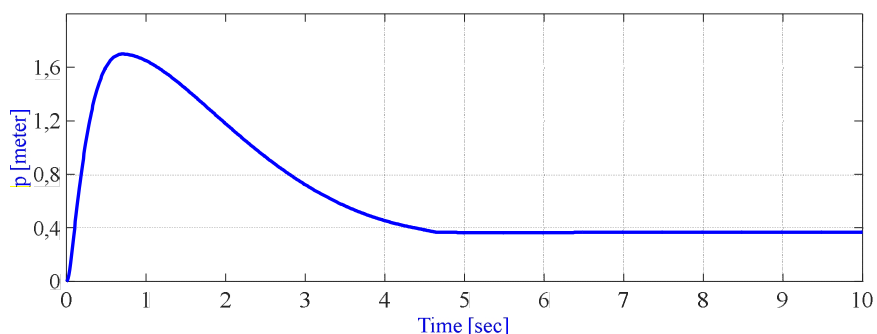

Fig. 10. Result of Robot Position Response Simulation with Initial Angle 0.2 radian and $\boldsymbol{p}_{\boldsymbol{d}}=0.4$ meter.

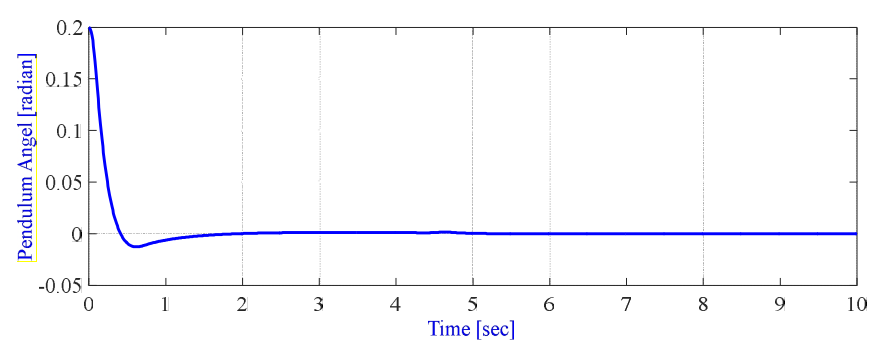

Fig. 11. Result of Pendulum Response Simulation with Initial Angle 0.2 radian and $p_{d}=0.4$ meter

\section{DISCUSSION}

The simulation result shows that the controller for two wheels inverted pendulum robot can stabilize pendulum angle in 0 radian and close to the desired position. The simulation results on fuzzy traveling and position control (FTPC) have an error of about $8 \%$ to control and stabilize the two-wheeled inverted pendulum robot in the desired position. Huang et al. developed the design and implementation of fuzzy control on a two-wheeled inverted pendulum and resulted similar results [10]. The combination of FTPC and FBSC works very well [15]. The proposed FTPC can enhance the performance of the FBSC. For the FBSC, the TWIP moves backward quickly to avoid falling. Then, the TWIP travels forward to reach the desired position due to the effort of the FTPC.

\section{CONCLUSION}

Based on the testing result of Mamdani and Takagi-Sugeno fuzzy control system simulation using Parallel Distributed Compensation (PDC) concept and pole placement technic in this research, it can be concluded that the simulation result of the FBSC controller is that the two wheels inverted pendulum robot system is able to maintain the upright pendulum position at angle 0 radian. Meanwhile, the FTPC controller simulation result is that the two wheels inverted pendulum robot system can approach the desired position with an error of about $8 \%$. This research can be developed by implementing hybrid fuzzy control on a two-wheeled pendulum robot.

\section{REFERENCES}

[1] Y.-W Liang, S.-D. Xu, D.-C Liaw, and C.-C Chen, "A Study of T-S model based SMC scheme with application to robot control”, IEEE Trans. Ind Electron., vol. 55, no.11, page 3964-3971, November 2008.

[2] Tanaka, K and T. Kosaki, "Design of a stable fuzzy controller for an articulated vehicle”, IEEE Trans. Syst., Man, Cybern B, Cybern., vol.27, no. 3, page 552-558, June 1997.

[3] Tanaka, K, M. Iwasaki and H. O. Wang, "Switcing control of an R/C hovercraft: stabilization and smooth Switcing”, IEEE Trans. Syst., Man, Cybern B, Cybern., vol.31, no. 6, page 853-863, December 2001.

[4] Tanaka, K, H. Ohtakeaki dan H. O. Wang, "A practical design approach to stabilization of a 3-DOF RC helicopter ", IEEE Trans. Control Syst. Technol., vol. 12, no. 2, page 315-325, March 2004.

[5] Ogata, Katsuhiko., "Teknik Kontrol Automatik", translated by Ir. Edi Leksono, Erlangga, Jakarta, 1993.

[6] A-M Almeshal, K-M Goher, "Robust hybrid fuzzy logic control of a novel two wheeled robotic vehicle with a movable payload under various operating conditions" IEEE, page 747-752, September 2012.

[7] Kharola, K "The control of Two-wheeled Inverted Pendulum Robot (TWIPR) using fuzzy logic", IEEE, December 2015.

[8] M-A Akmal, N-F Jamin and N-M Ghani, "Fuzzy Logic Controlle for Two Wheeled EV3 LEGO Robot” IEEE, page 134-138, Desember 2017.

[9] Huang, J, Ri, M, and Wu, D, "Interval Type-2 Fuzzy Logic Modeling and Control of a Mobile Two-Wheeled Inverted Pendulum" IEEE. Trans on Fuzzy System, October 2017.

[10] C.-H Huang, W-J Wang, and C-H Chiu, "Design and implementation of fuzzy control on a two wheel inverted pendulum", IEEE Trans. Ind Electron., vol. 58, no.7, July 2011.

[11] Johnson, Thomas, Siteng Zhou, Wei Cheah, Warren Mansell, Rupert Young, and Simon Watson. "Implementation of a perceptual controller

\section{Accredited by Ministry of Research and Technology /National Research and Innovation Agency, Indonesia Decree No: 200/M/KPT/2020


for an inverted pendulum robot." Journal of Intelligent \& Robotic Systems 99, no. 3 (2020): 683-692.

[12] Music, Zenit, Fabio Molinari, Sebastian Gallenmüller, Onur Ayan, Samuele Zoppi, Wolfgang Kellerer, Georg Carle, Thomas Seel, and Jörg Raisch. "Design of a networked controller for a two-wheeled inverted pendulum robot." IFAC-PapersOnLine 52, no. 20 (2019): 169-174.

[13] Wardana, Ananta Adhi, Takeshi Takaki, Mingjun Jiang, and Idaku Ishii. "Development of a single-wheeled inverted pendulum robot capable of climbing stairs." Advanced Robotics 34, no. 10 (2020): 674-688.

[14] Rigatos, Gerasimos, Krishna Busawon, Jorge Pomares, and Masoud Abbaszadeh. "Nonlinear optimal control for the wheeled inverted pendulum system." Robotica 38, no. 1 (2020): 29-47.

[15] Song, Qi, Qinglei Zhao, Shuxin Wang, Qiang Liu, and Xiaohe Chen. "Dynamic path planning for unmanned vehicles based on fuzzy logic and improved ant colony optimization." IEEE Access 8 (2020): 62107-62115.

Accredited by Ministry of Research and Technology /National Research and Innovation Agency, Indonesia Decree No: 200/M/KPT/2020 